\title{
Compact LCOS-SLM Based Polarization Pattern Beam Generator
}

\author{
Xuejie Zheng, Angel Lizana, Alba Peinado, Claudio Ramírez, Jose L. Martínez, Andrés Márquez, \\ Ignacio Moreno, Member, IEEE, and Juan Campos
}

\begin{abstract}
In this paper, a compact optical system for generating arbitrary spatial light polarization patterns is demonstrated. The system uses a single high-resolution liquid crystal (LC) on silicon (LCOS) spatial light modulator. A specialized optical mount is designed and fabricated using a 3D printer, in order to build a compact dual optical architecture, where two different phase patterns are encoded on two adjacent halves of the LCOS screen, with a polarization transformation in between. The final polarization state is controlled via two rotations of the Poincaré sphere. In addition, a relative phase term is added, which is calculated based on spherical trigonometry on the Poincaré sphere. Experimental results are presented that show the effectiveness of the system to produce polarization patterns.
\end{abstract}

Index Terms-Adaptive optics, displays, optical modulation, optical polarization, optical retarders, optical vortices, stokes parameters.

\section{INTRODUCTION}

$\mathbf{T}$ HE production of two-dimensional polarization patterns has become a relevant issue for a number of different applications. In particular, cylindrical vector beams [1], of which radially and azimuthally polarized beams are a subclass, are especially interesting due to their very appealing properties upon focalization with high aperture lenses [2], or because their relation with the orbital angular momentum of light [3]. Additionally, they are eigen-solutions of cylindrical resonators and optical fibers [4].

On the other hand, LC modulators are optoelectronic devices capable of modulating the state of polarization (SOP), which can be arranged in pixelated panels. LC devices have been developed with electrode patterns specific for the production of optical vortices and radially polarized beams [5]. However, their usual arrangement is as a rectangular grid of pixels, commonly known

Manuscript received November 25, 2014; revised January 2, 2015, January 8, 2015, and January 19, 2015; accepted January 19, 2015. Date of publication January 20, 2015; date of current version March 16, 2015. This work was supported by the Spanish Ministerio de Economia y Competitividad under Grants FIS2012-39158-C02-01 and FIS2012-39158-C02-02 and the Catalan Government contract 2014 SGR 1639.

X. Zheng, A. Lizana, A. Peinado, C. Ramírez and J. Campos are with the Department of Physics, Universitat Autònoma de Barcelona, 08193 Bellaterra, Spain (e-mail: xuejiezheng0603@gmail.com; Angel.Lizana@uab.cat; Alba.Peinado@uab.cat; cramirezxp@gmail.com; Juan.Campos@uab.cat).

A. Márquez is with the Department of Physics, Systems Engineering and Signal Theory, Universidad de Alicante, 03080 Alicante, Spain (e-mail: andres. marquez@ua.es).

J. L. Martínez and I. Moreno are with the Department of Materials Science, Optics and Electronics Technology, Universidad Miguel Hernández de Elche, 03202 Elche, Spain (e-mail: josel.martinezf@gmail.com; i.moreno@umh.es).

Color versions of one or more of the figrues in this paper are available online at http//ieeexplore.ieee.org.

Digital Object Identifier 10.1109/JLT.2015.2395256 as LC spatial light modulators (LCSLM). LCSLMs can be used to produce spatially variant SOP controlled from a computer and, in recent years, a variety of different methods and systems have been developed to create such $2 \mathrm{D}$ polarization patterns with these devices [6]-[21].

One general approach has been based on optical processing systems, where diffraction gratings are encoded onto a LCSLM, and different diffraction orders are filtered and their SOP are modified in the desired manner, before recombination [6]-[9]. Another related approach uses a computer-generated hologram displayed on a SLM within a Sagnac interferometer [10].

Other approaches use directly the LCSLM capability to modulate SOP as a function of the applied voltage [11]. Common parallel-aligned LCSLMs are devices intended to produce phase-only modulation. They act as linear retarders that can modulate only the electric field linear component parallel to the LC director. Therefore, in order to achieve a general SOP modulation, methods have been developed based on displaying independent control of two different halves of a SLM [12]-[16] or by using with two different SLMs [17]-[19]. These methods permit to control two parameters required to produce arbitrary SOPs. The different methods differ either in the parameters used to achieve SOP modulation, or in the optical architecture. But in all cases, the capability to separately encode unrelated patterns gives these systems much greater flexibility. Additional flexibility is obtained by adding a second LCSLM to incorporate independent amplitude and phase control over the SOP modulation [20], [21].

Nevertheless, in spite of this great recent advance in the field, all the proposed techniques are bulky systems and very sensitive to possible misalignments. In [22], [23], a compact system applied to demultiplex optical modes is proposed. It is based on a parallel aligned LCOS-SLM and the light steering is made by polarizing beam-splitters. The system works in the Fourier domain and modifies the phase of each polarization component. To control both, amplitude and phase in the spatial domain, they use a simulated annealing algorithm based diffractive optics technique. With this technique they evaluate what should be the phase distribution on the Fourier domain in order to control both amplitude and phase in a limited region of the spatial domain. Our aim is the development of a compact system capable of producing arbitrary SOP spatial distributions in the spatial domain, using in this way the full bandwidth of the LCOS-SLM.

Nowadays, the advances in LCOS-SLM technology provide devices with very large number of pixels and high definition and resolution panels with dimensions below centimeter scale. In addition $3 \mathrm{D}$ printing technology, combined with high quality 


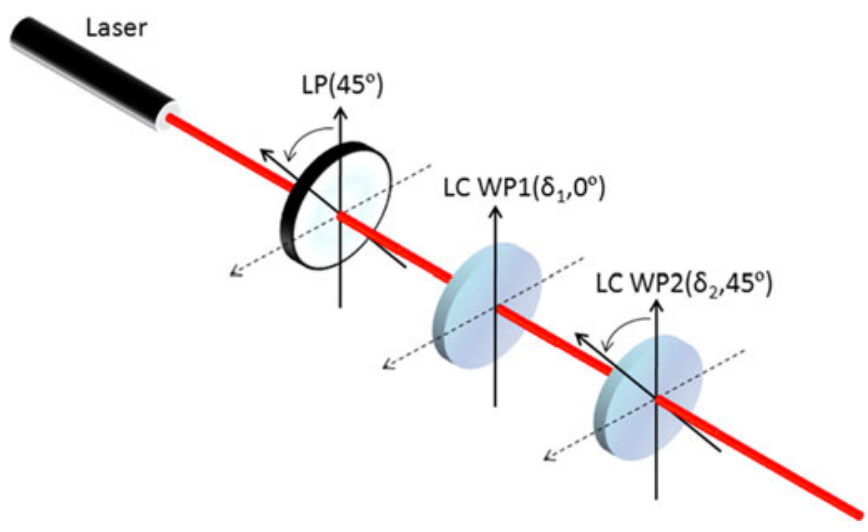

Fig. 1. PSG composed of two variable linear wave-plates.

optical components, can be used to fabricate very compact optical systems. This is the purpose of this work. Here we demonstrate a first prototype of a compact polarization pattern generator based on high resolution LCOS display, where the full system optical mount is developed using 3D printing technology and combined with high quality optical components. The system is capable of producing spatial patterns with complete control of the SOP modulation. It is based on inducing two consecutive independent polarization transformations that can be visualized to produce two independent rotations in the Poincaré sphere, in an equivalent manner to the system proposed in [17]. A residual phase modulation is produced, and can be analyzed in terms of the Pancharatman-Berry phase [24]. Experimental results are provided that demonstrate the polarization encoding. In a first stage, we applied the developed system to produce a versatile polarization state generator (PSG) with almost continuous variation of the selected SOP. Then, in a second stage, we demonstrate the production of cylindrical vector beams.

The paper is organized as follows. After this introduction, Section II describes the optical architecture and presents the developed optical 3D mount and optics. Then, Section III presents an analysis of the SOP modulation capability of the system, based on the Poincaré sphere transformations, including the calculation of the residual phase modulation. Section IV presents experimental results of different vectorial beams generated with the developed compact polarization pattern generator. Finally, Section V presents the conclusions of the work. For completeness, an Appendix is included at the end of the paper to analyze the proposed system with the Jones formalism, which shows equivalent results with the alternative derivation based on the Poincaré sphere transformations.

\section{COMPACT OpticAl ARCHITECTURE}

The architecture of the set-up we propose is based on the PSG shown in Fig. 1. It is based on the polarization state detector proposed in [25], but the elements are placed in opposite order. It is also equivalent to the system in Ref. [17]. It is composed of a linear polarizer oriented at $45^{\circ}$, a linear wave-plate with variable phase shift $\delta_{1}$ oriented at $0^{\circ}$ (LC-WP1), and a second linear wave-plate with variable phase shift $\delta_{2}$ oriented at $45^{\circ}$ (LC-WP2). As it will be shown in the next section, this system



Fig. 2. Scheme of the optical setup. LCOS-SLM indicates the display; HWP1 and HWP2 indicate half-wave plates with 22.5 relative angle; BS1 and BS2 are non-polarizing beam splitters; $L$ is a converging lens; $L B$ indicates a light barrier to block light. Red arrows indicate the trajectory of the modulated beam. Inset: Alternative set-up without the use of beam-splitters, based on the combination of three mirrors and one lens.

permits the generation of an arbitrary SOP by adjusting these two phase shifts.

However, with the idea to build a robust and compact experimental set-up, to decrease costs, and to be able to create arbitrary polarization patterns, we have reduced the number of elements to one single spatial light modulator. This is achieved by providing an adequate double pass of the light through a LCOS-SLM.

Fig. 2 shows a scheme of the optical setup. A collimated laser beam (He-Ne, $\lambda=632.8 \mathrm{~nm}$ ) is launched onto the system. It is linearly polarized, orientated at 45 degrees with respect to the SLM LC director axis (considered perpendicular to the page plane in Fig. 2). A first beam-splitter (BS1) divides the incident light. The transmitted beam is blocked by the light barrier (LB), while the reflected beam is directed onto one half of the LCOS-SLM screen, which is used to encode one first polarization transformation on the light beam. The beam is then reflected back to BS1, and the transmitted part passes through a system containing two prisms, a converging lens, a second beam-splitter (BS2) and two half-wave plates (HWP). In this set-up, the beam-splitters should be non-polarizing to preserve the polarization that is generated in each step. Therefore, in each pass through the beam-splitters BS1 and BS2, the light intensity is reduced to one half. In those applications where the intensity losses are especially critical, alternative systems should be devised. An alternative to avoid the use of beam splitters may consist in using oblique incidence on the SLM, but then, the system becomes larger. An example of oblique incident architecture can be seen in the inset of Fig. 2.

The two prisms (P1) and (P2) are included in the set-up to properly steer the light beam, leading to a compact architecture. A convergent lens (L) images the first half of the SLM over the second half of the screen, which encodes the second polarization transformation. The two half-wave plates (HWP1 
and HWP2, respectively), are oriented with a relative angle of $22.5^{\circ}$ between them. This specific combination of retarders acts as a polarization rotator with a rotation angle of $45^{\circ}$. This fact is very important since it ensures a system being equivalent to the one discussed in Fig. 1. (i.e., a combination of two Parallel Aligned (PA)-LCOS displays with a relative angle between their fast axes of $45^{\circ}$ ). Note, however, that the system in Fig. 2 is not entirely equivalent to the one in Fig. 1, since a final polarization rotation element would be required at the output. However, this polarization rotation simply implies a rotation of the reference frame at the final output beam.

As stated before, by achieving this construction, any fully polarized SOP can be generated by properly selecting the phase shifts $\delta_{1}$ and $\delta_{2}$ encoded onto each half of the LCOS-SLM.

The compact set-up sketched in Fig. 2 has been experimentally implemented. The beam-splitters and prisms are from Thorlabs (models BS010 and PS910H-A respectively). The lens L1 is also from Thorlabs, model LB1212-A, with a focal length of $20 \mathrm{~mm}$ and an aperture of $9 \mathrm{~mm}$. This lens focal length was selected to match a $2 f$ configuration in order to achieve a unity magnification (object distance is equal to image distance, equal to $2 f$ ). Finally, another converging lens was placed at the output of the system, to image the right part of the SLM onto a CCD camera.

Actually, the whole optical system, with the exception of the SLM, is placed in a box-holder about $20 \mathrm{~mm} \times 20 \mathrm{~mm} \times$ $20 \mathrm{~mm}$ (see Fig. 3), leading to a very compact set-up, and very robust system. The box holder was manufactured onto polylactide (PLA) thermoplastic polymer by means of a 3D-printer, RepRap BCN3D+. Fig. 3(a) shows the holder design created with Rhinoceros software.

Fig. 3(b) shows a picture of the fabricated system to be placed in front of the SLM. Two lateral screws were placed on the prism P1 wall, in order to achieve a fine lateral displacement in the reflected beam, so it can be properly centered on the rest of the system.

Finally, the LCOS-SLM is a parallel aligned (PA) display distributed by HoloEye, model PLUTO SLM. This is an active matrix reflective mode device with a rectangular screen with $1.8 \mathrm{~cm}$ diagonal $1920 \times 1080$ pixels with a pixel pitch of $8 \mu \mathrm{m}$ and $87 \%$ fill factor. The rectangular shape of this device is ideally suited for this architecture, since the screen can be divided in two nearly square halves.

\section{Polarization TRAnSFormations AND Phase MOdulation}

In this section we describe how the optical system presented above provides the desired SOP transformation in terms of the two-phase levels addressed to the two patterns in the LCOSSLM. There are different methods for this analysis. We present here the analysis in terms of the Mueller-Stokes matrix formalism, which more easily lends itself to physical insights of the polarization transformation through the related Poincaré sphere [26]. However, the Mueller-Stokes formalism does not provide directly information about the relative phase shift

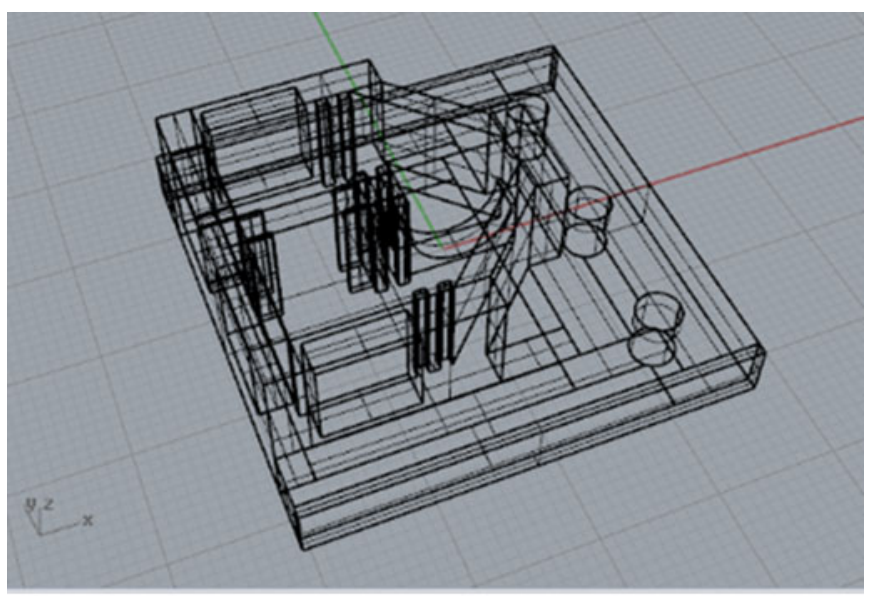

(a)

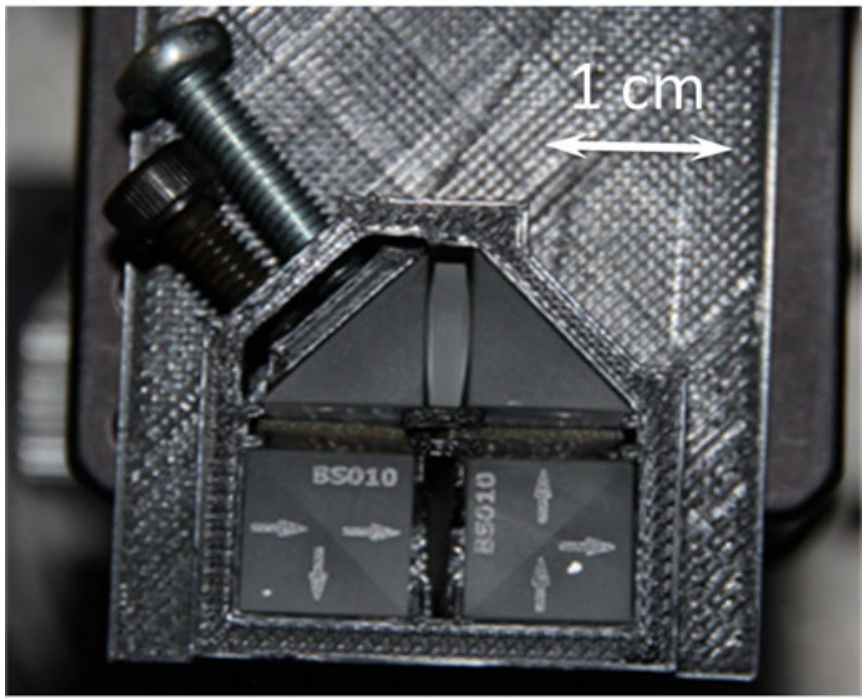

(b)

Fig. 3. (a) Design of the holder architecture, (b) fabricated experimental compact holder with optical elements.

modulation that accompanies this generated SOP transformation. In order to derive this additional information, we apply spherical geometry considerations on the Poincaré sphere [24]. However, for the sake of completeness, the Appendix section provides an alternative complete derivation in terms of the Jones matrix formalism.

\section{A. Analysis in Terms of Mueller-Stokes Formalism}

The Stokes parameters of elliptically polarized light with azimuth $\alpha$ and ellipticity $\varepsilon$ (see Fig. 4(a)) are given by [26]:

$$
S=\left(\begin{array}{c}
S_{0} \\
S_{1} \\
S_{2} \\
S_{3}
\end{array}\right)=\left(\begin{array}{c}
1 \\
\cos (2 \varepsilon) \cos (2 \alpha) \\
\cos (2 \varepsilon) \sin (2 \alpha) \\
\sin (2 \varepsilon)
\end{array}\right),
$$

where $S_{0}=1$ has been selected since we deal with fully polarized light. Additionally, there is a biunivocal correspondence 


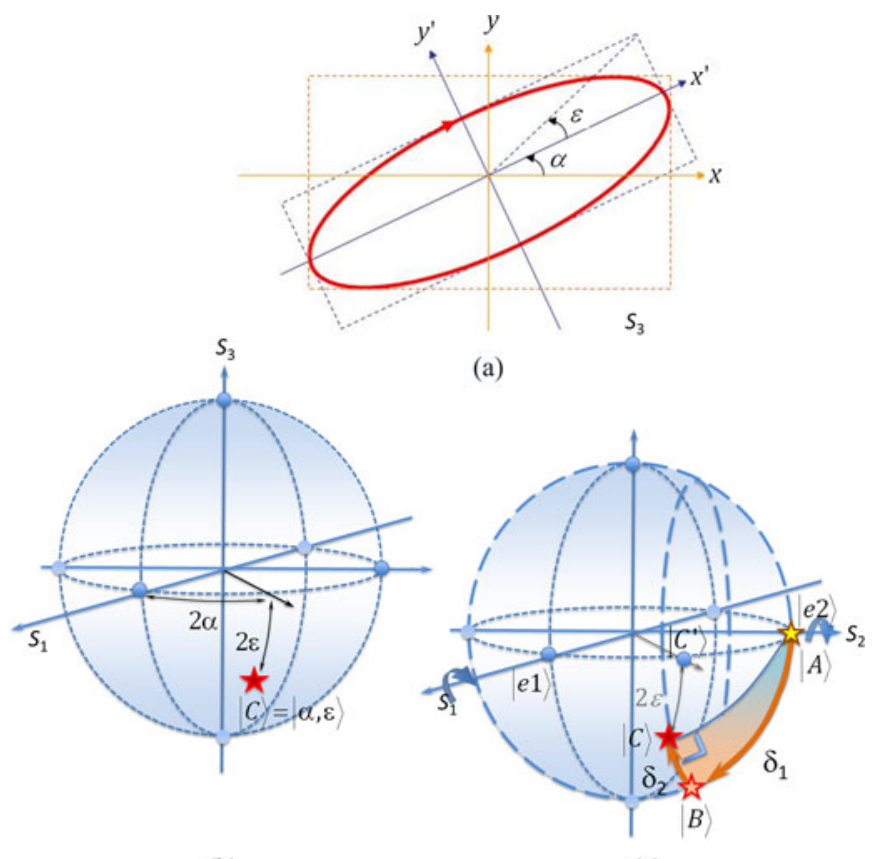

(b)

(c)

Fig. 4. (a) Representation of a SOP with ellipticity $(\varepsilon)$ and azimuth $(\alpha)$. (b) Representation on the Poincare sphere. (c) SOP transformations to produce a desired $(\varepsilon, \alpha)$ SOP with the proposed system.

between this SOP and a point $\mid C$ on the Poincaré sphere surface, with longitude $2 \alpha$ and latitude $2 \varepsilon$, respectively (see Fig. 4(b)).

The second relevant feature of the Poincaré sphere for our purpose is related to the action of a linear retarder with phase shift $\delta$ and orientation $\theta$, which can be visualized as a $\delta$-rotation of the incident SOP along the axis defined by $\theta$ [26]. Therefore, the polarization transformation produced by the system in Fig. 1 can be visualized as two Poincaré sphere rotations, as indicated in Fig. 4(c). We follow here the sign convention in [26], where a clockwise rotation corresponds to a positive phase-shift. We start with the linearly polarized state oriented at $45^{\circ}$, represented as point $\mid A$ in Fig. 4(c). This light traverses a first wave-plate oriented at $0^{\circ}$; therefore a first rotation of angle $\delta_{1}$ is produced around the $S_{1}$ axis, leading to the intermediate state $\mid B$. Finally, the passage of light though the second wave-plate, oriented at $45^{\circ}$, produces a second rotation of the sphere, now around the $\mathrm{S}_{2}$ axis, and an angle $\delta_{2}$, leading to the final state $\mid C$. Note that the complete surface of the sphere can be reached if the phase shifts $\delta_{1}$ and $\delta_{2}$ can be continuously tuned within the range $[0,2 \pi]$.

The Mueller-Stokes formalism directly provides relations between the phase shifts $\delta_{1}$ and $\delta_{2}$ and the ellipticity and azimuth angles of the generated SOP. The normalized output Stokes parameters, $S_{\text {out }}$, are given by the following relation [26]:

$$
S_{\text {out }}=D_{45}\left(\delta_{2}\right) \cdot D_{0}\left(\delta_{1}\right) \cdot L_{45}
$$

where $L_{45}=\left(\begin{array}{llll}1 & 0 & 1 & 0\end{array}\right)^{T}$ are the Stokes parameters of the initial linearly polarized state oriented at $45^{\circ}$, and $D_{0}$ and $D_{45}$ are the Mueller matrices corresponding to the linear wave-plates oriented at 0 and $45^{\circ}$, respectively, which take the following form [26]:

$$
D_{0}=\left(\begin{array}{cccc}
1 & 0 & 0 & 0 \\
0 & 1 & 0 & 0 \\
0 & 0 & c & s \\
0 & 0 & -s & c
\end{array}\right), D_{45}=\left(\begin{array}{cccc}
1 & 0 & 0 & 0 \\
0 & c & 0 & -s \\
0 & 0 & 1 & 0 \\
0 & s & 0 & c
\end{array}\right),
$$

where $s=\sin (\delta)$ and $c=\cos (\delta)$. Therefore, the matrix product in Eq. (2) leads to the following result:

$$
S_{\text {out }}=\left(\begin{array}{c}
1 \\
\sin \left(\delta_{1}\right) \sin \left(\delta_{2}\right) \\
\cos \left(\delta_{1}\right) \\
-\sin \left(\delta_{1}\right) \cos \left(\delta_{2}\right)
\end{array}\right) .
$$

By comparing Eqs. (1) and (4) the following relations are easily derived:

$$
\begin{aligned}
\tan (2 \alpha) & =\frac{1}{\tan \left(\delta_{1}\right) \sin \left(\delta_{2}\right)}, \\
\sin (2 \varepsilon) & =-\sin \left(\delta_{1}\right) \cos \left(\delta_{2}\right),
\end{aligned}
$$

that provide the $(\varepsilon, \alpha)$ parameters of the generated SOP as a function of the encoded phase shifts $\delta_{1}$ and $\delta_{2}$. The comparison of Eqs. (1) and (4) also directly provide inverse relations with the phase shifts $\delta_{1}$ and $\delta_{2}$ required to obtain a polarization ellipse with parameters $(\varepsilon, \alpha)$ :

$$
\begin{aligned}
\cos \left(\delta_{1}\right) & =\sin (2 \alpha) \cos (2 \varepsilon), \\
\tan \left(\delta_{2}\right) & =-\frac{\cos (2 \alpha)}{\tan (2 \varepsilon)} .
\end{aligned}
$$

However, although these relations completely define the SOP, there is no information about the relative phase shift modulation between different SOP generated with this system. This phase difference might be relevant especially in polarization diffractive elements. For that purpose, next we apply spherical trigonometry considerations. A complementary analysis based on the Jones matrix formalism is also presented in the Appendix section, to the sake of completeness.

\section{B. Analysis Based on Spherical Trigonometry on the Poincaré Sphere}

Following [24], SOP transformations and derivation of the coupled phase modulation can be obtained through the Poincaré sphere transformations described in Fig. 4(c). SOPs $|A|$,$B and$ $\mid C$ define a spherical triangle. The trajectory from $\mid A$ to $\mid B$ follows a geodesic arc with length $\delta_{1}$. On the contrary, trajectory from $\mid B$ to $\mid C$, with length $\delta_{2}$, does not follow a geodesic arc. The spherical triangle is closed with another geodesic arc that joints points $\mid C$ and $\mid A$, forming a rectangular angle at point $\mid C$. By applying the spherical cosine and sine laws to this spherical triangle, it is straightforward to derive the same relations as in Eqs. (5) and (6). In addition, the phase modulation related to these trajectories on the Poincaré sphere can also be derived by following the procedure described in Ref. [24]. This procedure 
indicates that when a general input SOP $\mid a$ is transformed onto an output SOP $\mid b$ by a waveplate with polarization eigenvectors $\mid e$ and $\mid e^{\prime}$, the relative phase shift $\varphi$ gained in this polarization transformation is given by

$$
\varphi=\phi_{e}+\frac{\Omega_{b e}}{2}-\frac{\Omega_{a e}}{2},
$$

where $\phi_{e}$ is the phase-shift gained by the waveplate eigenvector $\mid e$, and $\Omega_{b e}$ denotes the solid angle subtended by the spherical rectangle enclosed by the geodesic arc joining state $\mid b$ and the eigenstate $\mid e$, the equator line, and the two meridians passing through $\mid b$ and $\mid e$. Half this solid angle corresponds to the phase of the inner product $e \mid b$. This calculation can be done through any of the two eigenvectors of the wave-plate, which lie on antipodal points on the Poincaré sphere.

Let us first evaluate the phase gained at the intermediate point $\mid B$ with respect to the initial polarization (point $\mid A$ ). In this case, we consider the eigenstate located at the positive $S_{1}$ axis (denoted as $\mid e_{1}$ in Fig. 4(c)), which has a positive phase shift $\delta_{1}$. The two projections provide $\Omega_{A e 1}=0$ and $\Omega_{B e 1}=-\delta_{1} / 2$, where the sign convention in [24] is followed. Therefore, the total phase shift of this first step is directly $\varphi_{1}=\delta_{1} / 2$.

For the second step, from $\mid B$ to $\mid C$, we must consider that now the rotation is around $S_{2}$ axis. We select as the eigenvector the positive state along this axis, denoted as $\mid e_{2}$ in Fig. 4(c). Since $\mid B$ and $\mid e_{2}$ lie in the same meridian, now $\Omega_{B e 2}=0$. The calculation of $\Omega_{\mathrm{Ce} 2}$ must be done through the spherical triangle defined by $\mid e_{2}$, the final state $\mid C$, and the projection of this onto the equator, labeled as point $\mid C^{\prime}$ in Fig. 4(c). The segment from $\mid C$ to $\mid C^{\prime}$ is equal to $2 \varepsilon$, the segment from $\mid C^{\prime}$ to $\mid e_{2}$ is $90^{\circ}-2 \alpha$, and the segment from $\mid C$ to $\mid e_{2}$ is $\delta_{1}$. And the angle at point $\mid e_{2}$ is equal to $90^{\circ}-\delta_{2}$. As a result, and applying spherical trigonometry relations, the following expression can be derived to the solid angle of this triangle:

$$
\tan \Omega_{C e 2}=\frac{\sin ^{2}\left(\frac{\delta_{1}}{2}\right) \sin \left(2 \delta_{2}\right)}{1-2 \sin ^{2}\left(\frac{\delta_{1}}{2}\right) \cos ^{2}\left(\delta_{2}\right)} .
$$

Therefore, the total phase shift gained at this second transformation is $\varphi_{2}=\delta_{2}+\Omega_{C e 2} / 2$, and the total phase shift from $\mid A$ to $\mid C$ is:

$$
\begin{aligned}
\varphi & =\frac{\delta_{1}}{2}+\delta_{2}+\frac{\Omega_{C e 2}}{2} \\
& =\frac{\delta_{1}}{2}+\delta_{2}+\frac{1}{2} \arctan \left(\frac{\sin ^{2}\left(\frac{\delta_{1}}{2}\right) \sin \left(2 \delta_{2}\right)}{1-2 \sin ^{2}\left(\frac{\delta_{1}}{2}\right) \cos ^{2}\left(\delta_{2}\right)}\right) .
\end{aligned}
$$

This equation can be rewritten as: $\varphi=\frac{1}{2}\left(\delta_{1}+\delta_{2}\right)+$ $\frac{1}{2}\left(\Omega_{C e 2}+\delta_{2}\right)$, which after some trigonometric manipulation, leads to the following simpler expression:

$$
\varphi=\frac{\delta_{1}+\delta_{2}}{2}+\frac{1}{2} \arctan \left(\frac{\tan \left(\delta_{2}\right)}{\cos \left(\delta_{1}\right)}\right) .
$$

This equation provides the relative phase shift between the different SOP generated with the proposed setup, as a function of the two required phase shifts $\delta_{1}$ and $\delta_{2}$.

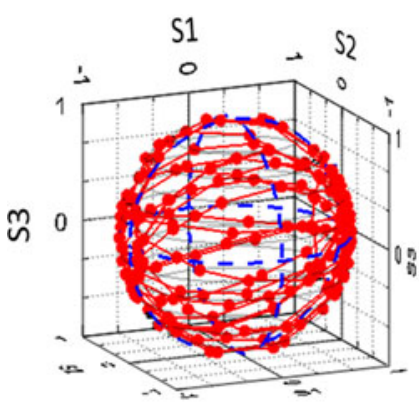

(a)
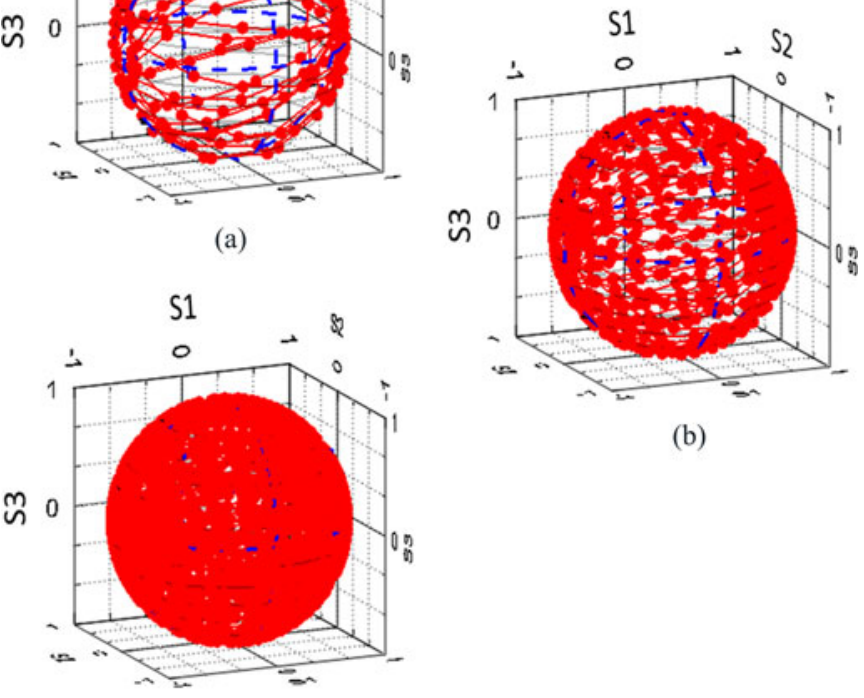

(b)

(c)

Fig. 5. Available SOP generated with uniform screens with gray level steps of: (a) 20, (b) 10, and (c) 5. Therefore, $14^{2}=196,27^{2}=729$, and $52^{2}=2704$ different SOP are available, respectively, uniformly distributed on the Poincaré sphere.

\section{EXPERIMENTAL RESULTS}

In order to test the optical system, we first analyzed its performance as a PSG by addressing screens of uniform gray level to each half of the LCOS-SLM. The gray level can be adjusted to values in the range $(0,255)$, each one corresponding to a different phase shift on the LCOS-SLM. The display is configured to provide a $2 \pi$ phase modulation in the complete range of addressed gray levels (from 0 to 255 gray level). Different combinations of pairs of gray levels $\left(g_{1}, g_{2}\right)$ have been selected to be addressed to each half of the LCOS screen, and the generated SOPs at the output have been tested by measuring the corresponding Stokes vector with a commercial Stokes polarimeter.

Fig. 5 shows the polarimeter results. In Fig. 5(a) we have first used a gray level step of 20, i.e., $g_{1}$ and $g_{2}$ have been assigned values $0,20,40, \ldots, 220,240,255$. Thus, the number of combinations is $14^{2}$ providing 196 different polarization states uniformly distributed in the Poincaré sphere. Each red spot represents a different generated SOP detected with the polarimeter. They show an excellent uniform distribution on the Poincaré sphere surface, although some SOP discretization is still clearly visible due to the large gray level selected step.

In Fig. 5(b) and (c) the gray level step was reduced to 10 and 5 respectively, leading to a total of 729 and 2704 different states of polarization respectively. Note that the Poincaré sphere is uniformly and completely filled with SOP generated with the system. In the limit, using all possible gray levels, the number of SOP would be $256^{2}=65536$ leading to practically continuous variation of the SOP. 

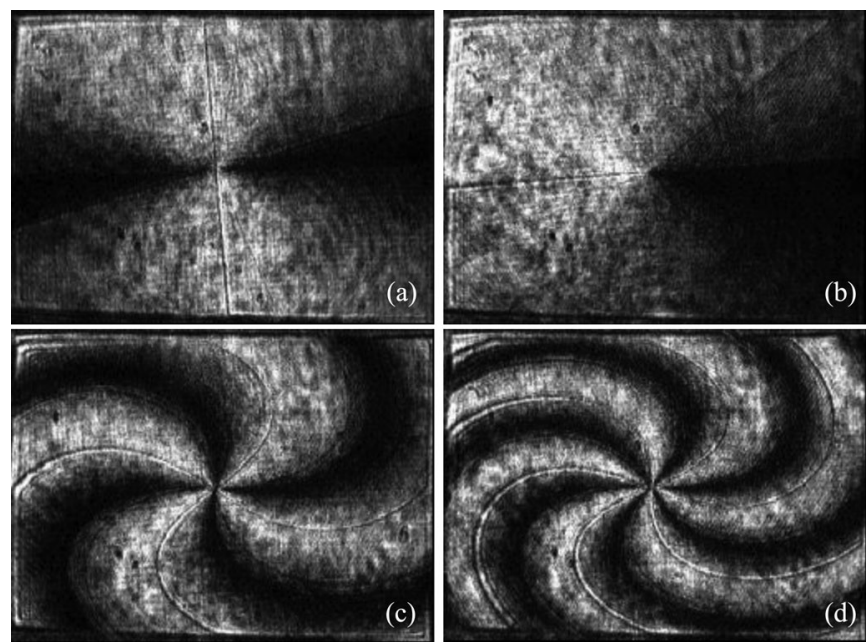

Fig. 6. CCD capture for different polarization distributions. A polarizer is placed in front of the CCD camera, oriented at 0 degrees. (a) Radial polarization (order $m=1$ ). (b) Order $m=0.5$. (c) Order $m=2$ plus quadratic phase shift $(d=0.5)$, and (d) Order $m=3$ plus quadratic phase shift $(d=1)$. Video files are attached that show the SOP variation by rotating the analyzer in front of the $\mathrm{CCD}$ detector.

A second test is performed for producing non-uniform distributions of polarization. The results are shown in Fig. 6. In order to visualize the corresponding polarization pattern, an analyzer is placed just in front of the CCD camera in Fig. 2. In the images shown in Fig. 6, this analyzer is oriented at $0^{\circ}$ (laboratory vertical direction). In the corresponding related videos, the analyzer is rotated and it can be seen that the whole intensity pattern performs the corresponding rotation, indicating the polarization angle.

We started by generating a light beam with radial distribution of linear polarization. In an ideal case, the radial polarization could be generated by encoding a spiral phase shift $\delta_{1}$, while encoding a uniform $\delta_{2}=\pi / 2$, in order to emerge linear states. However, due to experimental imperfections of the optical elements in the set-up (see Fig. 3) with respect to the theoretical values (caused by non-zero retardance values of the beam-splitters [27], certain experimental offset for the waveplates orientation, time-fluctuations of the phase introduced by the LCOS display [28], etc.), the above mentioned phase encoding procedure may lead to slightly modified results. To take into account those experimental deviations and thus, to achieve the best phase encoding, a searching software-based procedure is applied. The software, implemented on LabView, is based on the experimental calibration given in Fig. 5, where the relation between pairs of gray levels and the corresponding SOP measured is obtained. In fact, from this gray level pairsSOPs database, the implemented searching method provides the pair of gray levels related to the experimental SOP providing the minimum mean squared error when compared with the desired SOP. Note that once the relation between gray level pairs and SOP is known, the relation between the phases $\delta_{1}$ and $\delta_{2}$ and the gray level pair addressed to the LCOS display is directly obtained by using Eqs. (6).

Fig. 6(a) shows the experimental output beam measured on the CCD when generating a light beam with radial distribution

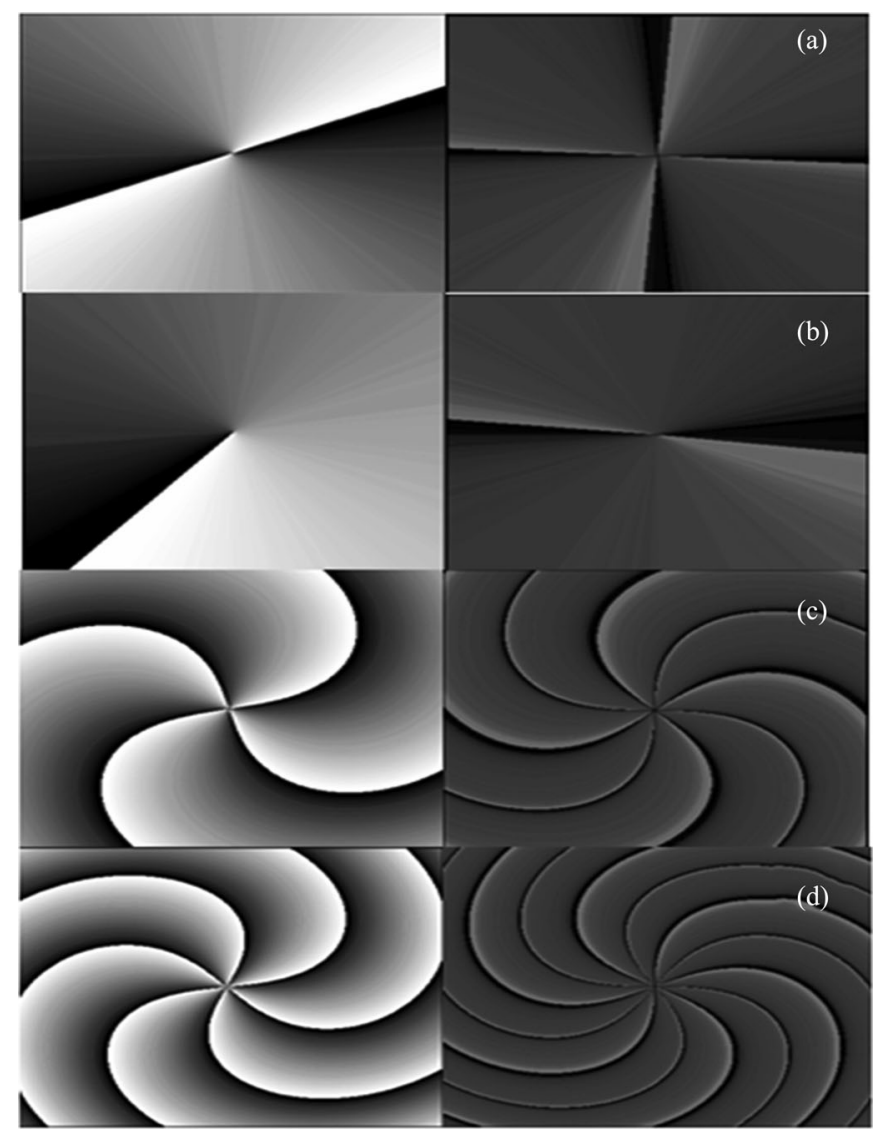

Fig. 7. Gray level distribution addressed to the first half (first column images) and to the second half (second column images) of the LCOS screen. (a) Radial polarization $(m=1, d=0)$. (b) Azimuthal polarization $(m=0.5, d=0)$. (c) Radial quad ( $m=2 ; d=0.5)$, and (d) Radial quad ( $m=3 ; d=1$ ).

of linear polarization. In addition, the dual gray level pattern encoded on the LCOS-SLM that generates this beam is shown in Fig. 7(a). Since the analyzer transmission axis is oriented at $0^{\circ}$, the radial distribution of linear SOP is observed as an angular progression from a maximum vertical intensity line (at $0^{\circ}$, vertical direction) to the null horizontal intensity line (at $90^{\circ}$, horizontal direction). Verification of the radial polarization is observed in the related videos, where the intensity pattern is reversed when the analyzer is rotated by $90^{\circ}$.

Fig. 6(b)-(d) show other spatially polarization variant beams. These SOP patters are created by displaying the images in Fig. 7(b)-(d) on the LCOS-SLM. Such patterns are given by a radial distribution with an additional quadratic phase shift pattern, as provided by the following relation:

$$
\delta=m \theta+d \pi r^{2},
$$

where $r$ is the radius and $\theta$ is the azimuthal angle, $m$ provides the number of whole rotations versus $\theta$ and $d$ is a parameter that determines the curvature of quadratic phase term. This is the retardance pattern to be addressed to the first half of the LCoS, i.e. $\delta_{1}(r, \theta)$, whereas to the second half a constant retardance value $\delta_{2}=\pi / 2$ must be addressed. Note that Fig. 7 highlights the necessity of using the SOP searching algorithm to accurately encode the desired SOP spatial distribution: for example, 
the second half of the LCoS display should ideally have a uniform gray level, but actually the appropriate gray level image to produce the desired results is not uniform.

In Fig. 6(b), only half azimuthal polarization rotation is produced on the beam $(m=1 / 2)$, and the quadratic phase term is avoided $(d=0)$. As a consequence, polarization is linear and vertical on left horizontal line, and it is linear and horizontal on right horizontal line, and it progressively rotates in between. Therefore, when this polarization distribution is projected over a linear polarizer fixed at $0^{\circ}$ of the vertical, the obtained intensity pattern presents a continuous intensity variation from a maximum intensity line at the left, to a null intensity line at the right.

Finally, Fig. 6(c) and (d) show the result when a radial quadratic distribution is encoded on the LCOS display. As it was shown in [16], this phase distribution produces a spiral linear SOP pattern. The number of dark and bright lines directly depends on the $m$ value (i.e., the distribution order) of the encoded spiral phase, and the helices curvature grows with $d$. These experimental results confirm the theory.

A final remark refers to the gray level distributions in Fig. 7 that produce the patterns in Fig. 6 (obtained through the SOP searching algorithm software). Note that the polarization distribution exiting from the first half of the LCOS screen is not only modified by the optical elements present in the compact optical system given in Fig. 3 but is also affected by an image inversion, due to the reflections present in the optical path from one half of the LCOS display to the other half. To compensate this effect, the gray level distribution addressed to the second half is inverted as well, as can be seen in the second column of Fig. 7.

\section{CONClusion}

In summary, we have developed a compact optical system based on a parallel-aligned LCOS-SLM that acts as a pixelated PSG, suitable for the production of complex spatial polarization patterns. The system can be regarded as the combination of two variable retarders with tunable retardance, with a relative orientation of $45^{\circ}$ among them. Although the system introduces some important losses, mainly due to the four passages through the beam splitters, it has been experimentally validated to act as a polarization generator covering the complete Poincaré sphere.

A complete analysis of the SOP modulation capability of the system has been presented, based on the Mueller-Stokes formalism. Additionally, the coupled relative phase modulation has also been derived based on spherical trigonometry relations on the Poincaré sphere. These relations have been alternatively derived using the Jones formalism in the Appendix, therefore providing an alternative point of view.

Based on the excellent results obtained, the suitability of the system to generate different spatially variant distributions of polarization has been validated.

\section{APPENDIX}

The analysis presented in the paper, based on the MuellerStokes formalism, provides a complete description of the SOP transformations of the system. In addition, the analysis of the Poincaré sphere transformation additionally provides information about the coupled relative phase modulation. However, many users are more familiar with the Jones formalism. The purpose of this Appendix is to provide an alternative derivation based on the Jones formalism, in order to complete the different views of the analysis.

The Jones formalism is not directly related to the azimutellipticity parameterization of the SOP. On the contrary, it is directly related to the complex amplitude components of the electric field, and therefore it can provide information about the relative phase shift between different SOP generated with the proposed system.

The same polarization transformation in Eq. (2) can be applied in the Jones formalism, but then the input normalized Jones vector is $\bar{L}_{45}=(1 / \sqrt{2} 1 / \sqrt{2})^{T}$ and the Jones matrices corresponding to the wave-plates are given by:

$$
\bar{D}_{0}=\left(\begin{array}{cc}
e^{i \delta} & 0 \\
0 & 1
\end{array}\right), \bar{D}_{45}=\bar{R}(-45) \cdot \bar{D}_{0} \cdot \bar{R}(+45),
$$

where the rotation matrix is

$$
R(\theta)=\left(\begin{array}{ll}
\cos \theta & \sin \theta \\
-\sin \theta & \cos \theta
\end{array}\right)
$$

Note that in Eq. (A1), the phase shift in $\bar{D}_{0}$ is limited to the $x$ component, while it is considered constant for the $y$ component, in agreement with the $x$-axis orientation of the LC director of the LCOS-SLM display.

The calculation of Eq. (2) by applying the Jones matrices in Eq. (A1) leads to the following result:

$$
\bar{J}_{\text {out }}=\frac{e^{i \bar{\delta}}}{\sqrt{2}}\left(\begin{array}{c}
\cos \Delta+i \sin \bar{\delta} \\
\cos \bar{\delta}-i \sin \Delta
\end{array}\right)
$$

where $\bar{\delta}$ and $\Delta$ are given respectively by,

$$
\bar{\delta}=\frac{\delta_{1}+\delta_{2}}{2}, \Delta=\frac{\delta_{1}-\delta_{2}}{2} .
$$

Note that the Jones vector does not provide a direct relation with the ellipticity-azimut parameterization of the polarization ellipse. This can be obtained by noting that the normalized Jones vector can be written in terms of $\alpha$ and $\varepsilon$ as [24]:

$$
\begin{aligned}
\bar{J}(\alpha, \varepsilon) & =\bar{R}(-\alpha) \cdot\left(\begin{array}{l}
\cos \varepsilon \\
i \sin \varepsilon
\end{array}\right) \\
& =\left(\begin{array}{c}
\cos \alpha \cos \varepsilon-i \sin \alpha \sin \varepsilon \\
\sin \alpha \cos \varepsilon+i \cos \alpha \sin \varepsilon
\end{array}\right) .
\end{aligned}
$$

By comparison of the Jones vector in Eq. (A3) with an output Jones vector $\bar{J}_{\text {out }}=e^{i \varphi} \bar{J}(\alpha, \varepsilon)$, Eqs. (5) and (6) derived with the Mueller approach can also be retrieved. But now, also the relative phase modulation between different SOPs can be derived.

By calculating $\bar{J}_{\text {out }}^{T} \cdot \bar{J}_{\text {out }}$, denoting $T$ the transposed Jones vector, the following equality is obtained for the vector in 
Eq. (A3) and for the vector $\bar{J}_{\text {out }}=e^{i \varphi} \bar{J}(\alpha, \varepsilon)$ :

$$
\begin{aligned}
\bar{J}_{\text {out }}^{T} \cdot \bar{J}_{\text {out }} & =e^{i 2 \bar{\delta}}\left(\cos \delta_{1} \cos \delta_{2}+i \sin \delta_{2}\right) \\
& =\cos (2 \varepsilon) e^{i 2 \varphi} .
\end{aligned}
$$

This complex values equality directly provides Eq. (5b) when the modulus is considered. And, when dealing with the phase values, the following relation is obtained:

$$
\varphi=\bar{\delta}+\frac{1}{2} \arctan \left(\frac{\tan \delta_{2}}{\cos \delta_{1}}\right) .
$$

This is exactly the same phase modulation (Eq. (10)) derived with the Poincaré sphere technique in Section III-B.

\section{ACKNOWLEDGMENT}

The authors would like to thank Jordi Prat-Camps for the manufacturing of the compact holder present in the optical set-up.

\section{REFERENCES}

[1] Q. Zhan, "Cylindrical vector beams: From mathematical concepts to applications," Adv. Opt. Photon., vol. 1, pp. 1-57, 2009.

[2] S. Quabis, R. Dorn, M. Eberler, O. Glöckl, and G. Leuchs, "Focusing light into a tighter spot," Opt. Commun., vol. 179, pp. 1-7, 2000.

[3] J. P. Torres, "Quantum engineering of light," Opt. Pure Appl., vol. 44, pp. 309-314, 2011.

[4] S. Ramachandran, P. Kristensen, and M. F. Yan, "Generation and propagation of radially polarized beams in optical fibers," Opt. Lett., vol. 34 pp. 2525-2527, 2009.

[5] J. Albero, P. Garcia-Martinez, N. Bennis, E. Oton, B. Cerrolaza, I. Moreno, and J. A. Davis, "Liquid crystal devices for the reconfigurable generation of optical vortices," J. Lightw. Technol., vol. 30, no. 18, pp. 3055-3060, Sep. 2012.

[6] X. L. Wang, J. Ding, W. J. Ni, C.-S. Guo, and H. T. Wang, "Generation of arbitrary vector beams with a spatial light modulator and a common path interferometric arrangement," Opt. Lett., vol. 32, pp. 3549-3551, 2007.

[7] X. L. Wang, Y. Li, J. Chen, C.-S. Guo, J. Ding, and H. T. Wang, "A new type of vector fields with hybrid states of polarization," Opt. Exp., vol. 18, pp. 10786-10795, 2010.

[8] J. Xin, C. Gao, C. Li, and Z. Wang, "Generation of polarization vortices with a Wollaston prism and an interferometric arrangement," Appl. Opt., vol. 51, pp. 7094-7097, 2012.

[9] I. Moreno, C. Iemmi, J. Campos, and M. J. Yzuel, "Jones matrix treatment for optical Fourier processors with structured polarization," Opt. Exp., vol. 19, pp. 4583-4594, 2011

[10] S. Liu, P. Li, T. Peng, and J. Zhao, "Generation of arbitrarily spatially variant polarization beams with trapezoid Sagnac interferometer," Opt. Exp., vol. 20, pp. 21715-21721, 2012.

[11] J. A. Davis, D. E. McNamara, D. M. Cottrell, and T. Sonehara, "Twodimensional polarization encoding with a phase-only liquid-crystal spatial light modulator," Appl. Opt., vol. 39, pp. 1549-1554, 2000.

[12] C. Maurer, A. Jesacher, S. Fürhapter, S. Bernet, and M. Ritch-Marte, "Tailoring of arbitrary optical vector beams," New J. Phys., vol. 9, pp. 78, 2007.

[13] D. Preece, S. Keen, E. Botvinick, R. Bowman, M. Padgett, and J. Leach, "Independent polarisation control of multiple optical traps," Opt. Exp., vol. 16, pp. 15897-15902, 2008.

[14] I. Moreno, J. A. Davis, T. Hernandez, D. M. Cottrell, and D. Sand, "Complete polarization control of light from a liquid crystal spatial light modulator," Opt. Exp., vol. 20, pp. 364-376, 2012.

[15] J. H. Clegg and M. A. A. Neil, "Double pass, common path method for arbitrary polarization control using a ferroelectric liquid crystal spatial light modulator," Opt. Lett., vol. 38, pp. 1043-1045, 2013.

[16] I. Moreno, J. A. Davis, D. M. Cottrell, and R. Donoso, "Encoding highorder cylindrically polarized light beams," Appl. Opt., vol. 53, pp. 5493$5501,2014$.
[17] F. Kenny, D. Lara, O. G. Rodríguez-Herrera, and C. Dainty, "Complete polarization and phase control for focus-shaping in high-NA microscopy," Opt. Exp., vol. 20, pp. 14015-14029, 2012.

[18] D. Maluenda, I. Juvells, R. Martínez-Herrero, and A. Carnicer, "Reconfigurable beams with arbitrary polarization and shape distributions at a given plane," Opt. Exp., vol. 21, pp. 5424-5431, 2013.

[19] C. S. Guo, Z. Y. Rong, and S. Z. Wang, "Double-channel vector spatial light modulator for generation of arbitrary complex vector beams," Opt. Lett., vol. 39, pp. 386-389, 2014.

[20] E. H. Waller and G. von Freymann, "Independent spatial intensity, phase and polarization distributions," Opt. Exp., vol. 21, pp. 28167-28174, 2013.

[21] W. Han, Y. Yang, W. Cheng, and Q. Zhan, "Vectorial optical field generator for the creation of arbitrarily complex fields," Opt. Exp., vol. 21, pp. 20692-20706, 2013.

[22] J. Carpenter, B. C. Thomsen, and T. D. Wilkinson, "Degenerate modegroup division multiplexing," J. Lightw. Technol., vol. 30, no. 24, pp. 3946-3952, Dec. 2012.

[23] J. Carpenter, B. J. Eggleton, and J. Schröder, "110 × 110 optical mode transfer matrix inversion," Opt. Exp., vol. 22, pp. 96-101, 2013.

[24] J. L. Martínez-Fuentes, J. Albero, and I. Moreno, "Analysis of optical polarization modulation systems through the Pancharatnam connection," Opt. Commun., vol. 285, pp. 393-401, 2012.

[25] A. Peinado, A. Lizana, J. Vidal, C. Iemmi, and J. Campos, "Optimization and performance criteria of a Stokes polarimeter based on two variable retarders," Opt. Exp., vol. 18, pp. 9815-9830, 2010.

[26] D. Goldstein, Polarized Light, 2nd edition, New York, NY, USA: Marcel Dekker Inc., 2003, pp. 65-86.

[27] A. Lizana, N. Martín, M. Estapé, E. Fernández, I. Moreno, A. Márquez, C. Iemmi, J. Campos, and M. J. Yzuel, "Influence of the incident angle in the performance of liquid crystal on silicon displays," Opt. Exp., vol. 17, pp. 8491-8505, 2009.

[28] A. Lizana, I. Moreno, A. Márquez, C. Iemmi, E. Fernandez, J. Campos, and M. J. Yzuel, "Time fluctuations of the phase modulation in a liquid crystal on silicon display: Characterization and effects in diffractive optics," Opt. Exp., vol. 26, pp. 16711-16722, 2008.

Xuejie Zheng received the M.Sc. degree in optoelectronic information engineering at the Huazhong University of Science and Technology, Wuhan, China, in 2011, and the M.S. degree in nanophotonics at the Université de Strasbourg, Strasbourg, France, in 2014.

He has conducted a research internship at the Autonomous University of Barcelona, Barcelona, Spain, in 2014, with a total duration of six months, where he has been developing new optical techniques, based on liquid crystal technology, to generate customized polarization distributions.

His research interests include the study of nanophotonics applications, the use of liquid crystal displays in diffractive optics applications and optoelectronics.

Angel Lizana received the M.Sc. degree in physics and the Ph.D. degree in physics at the Autonomous University of Barcelona, Barcelona, Spain, in 2006 and 2011, respectively.

His research interests include liquid crystal displays and their application to diffractive optics, as well as the design and implementation of polarimeters. He has been a postdoctoral scientist in the Laboratoire de Physique des Interfaces et des Couches Minces (LPICM) of the École Polytechnique (France), in 2011-12 and in 2013-2014, where he has worked in the optimization and implementation of a polarimeter able to operate in the infrared spectrum, and in the analysis of polarimetric information for its application to materials characterization. $\mathrm{He}$ is a postdoctoral researcher at the Autonomous University of Barcelona since 2014. He is a Member of the SPIE and of SEDOPTICA.

Alba Peinado received the B.S. degree in physics from the Autonomous University of Barcelona, Barcelona, Spain, in 2009, and the M.S. degree in photonics from Universitat Politècnica de Catalunya, Catalonia, Spain, in 2010. She is currently working toward the Ph.D. degree in physics at the Autonomous University of Barcelona.

Her research interests include polarimetry, including design, optimization and error analysis of polarimeters, as well as liquid crystal displays and their application to polarimeter architecture design and diffractive optics. She has been a Visiting Research Scholar at University of Connecticut, Storrs, CT, USA, for four months and at University of Arizona, Tucson, AZ, USA, for six months in 2012 and 2013, respectively. 
Claudio Ramírez received the B.S. degree in electronics engineering at Iberoamericana University, Mexico City, México, in 1995. He received the M.Sc. and Ph.D. degrees in Optical Sciences at Centro de Investigaciones en Óptica, Leon, México, in 2004 and 2011, respectively.

He was an invited professor of optics in 2012, and later he made a postdoctoral stay at Autonomous University of Barcelona, Barcelona, Spain, in 2014. His research interests include interferometry and polarization devices and its applications in image quality evaluation. He is a member of the OSA and SPIE.

Jose L. Martínez received the Telecommunication Engineering and Ph.D. degrees from University Miguel Hernández of Elche, Elche, Spain, in 2007 and 2012, respectively.

His research interest includes the characterization and use of Spatial Light Modulators in optical systems, as well as the development of electronic systems, programs and analysis techniques related to such devices and systems. His interests include digital holography systems and electronics. He was granted by the Valencian Government as postdoctoral researcher in the same university.

Andrés Márquez received the M.Sc. and Ph.D. degrees in physics from the Autonomous University of Barcelona, Barcelona, Spain, in 1997 and 2001, respectively.

He joined the Universidad de Alicante, Alicante, Spain, in 2000, where he is currently an Associate Professor of applied physics. His research interests include holographic recording materials, liquid crystal spatial light modulators, optical image processing and diffractive optics.
Ignacio Moreno received the B.S. degree in physics and the Ph.D. degree in physics at the Autonomous University of Barcelona, Barcelona, Spain, in 1992 and 1996, respectively.

His research interests include liquid crystal displays and its applications in diffractive and polarization optics. He has been a guest scientist at various international research centers like the Department of Physics at San Diego State University, San Diego, CA, USA, the FEMTO Institut at the Universite de Franche-Comté, Besançon, France, and the Departamento de Física at the Universidad de la Frontera, Temuco, Chile. He is currently the Director of the Doctoral Program on Industrial and Communications Engineering at University Miguel Hernandez of Elche, Elche, Spain. He was the Editor of the journal Optica Pura y Aplicada (OPA), edited by the Spanish Optical Society (SEDOPTICA), from 2005 to 2014, and he is currently an Associate Editor of the journal Optical Engineering. He is a Member of IEEE, OSA, SPIE and SEDOPTICA. He is SPIE Fellow Member and OSA Senior Member.

Juan Campos received the MSc degree in physics at the University of Zaragoza, Zaragoza, Spain, in 1981 and the Ph.D. degree at the Autonomous University of Barcelona, Barcelona, Spain, in 1986.

From 1982 to 1992, he has been professor of Optics at the University of Barcelona, Barcelona. Since 1993, he has been a Professor of Optics at the Autonomous University of Barcelona. Since 2005, he has been a Full Professor.

$\mathrm{He}$ has worked in the field of image quality evaluation, dealing with the influence of aberrations and apodization. He has also been working on optical pattern recognition, designing different kinds of filters by means of computergenerated holograms, introducing the color information, and by using spatial light modulators. He is studying the use of liquid crystal spatial light modulators in diffractive optics, and in polarimetry. He is working in algorithms to recover the shape from deflectometry measurements and interferometry. He is Fellow of the SPIE, OSA, and EOS. 